Check for updates

Cite this: RSC Adv., 2019, 9, 620

Received 19th September 2018 Accepted 2nd December 2018

DOI: $10.1039 / c 8 r a 07804 a$

rsc.li/rsc-advances

\title{
A water-stable luminescent metal-organic framework for effective detection of aflatoxin B1 in walnut and almond beverages $\uparrow$
}

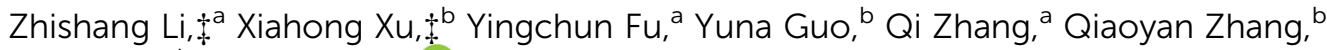 \\ Hua Yang*b and Yanbin Li (iD *ac
}

\begin{abstract}
Sensitive and rapid detection of aflatoxin B1 (AFB1) without using antibody or biomolecular modifications in water is achieved using a novel water-stable luminescent metal-organic framework (LMOF) termed $\mathrm{Zr}$ CAU-24. The 1,2,4,5-tetrakis(4-carboxyphenyl) benzene $\left(\mathrm{H}_{4} \mathrm{TCPB}\right)$-based LMOF with high water-stability has demonstrated drastic fluorescence fading in the presence of AFB1. The detection limit for AFB1 using this porous nanomaterial reaches as low as $19.97 \mathrm{ppb}(64 \mathrm{nM})$, which is below the applicable action level for peanut and corn products set by the FDA and among the most sensitive sensors reported for AFB1. We further investigated its response to five other mycotoxins including AFB2, AFG1, AFG2, AFM and OTA and their Stern-Volmer quenching efficiencies are significantly below that of AFB1 $\left(138461 \mathrm{M}^{-1}\right)$. The prepared water-stable LMOF was directly used for the detection of AFB1 in spiked walnut and almond beverages. High recovery rates (91-108\%) were achieved in $5 \mathrm{~min}$. We found that the quenching of $\mathrm{H}_{4}$ TCPB molecules towards mycotoxins was remarkably enhanced by anchoring them into the periodic framework and its mechanism was discussed. The presented method with acceptable detection limit is of potential for the development of low-cost, robust and sensitive sensors for the rapid detection of AFB1 in agricultural and food products.
\end{abstract}

\section{Introduction}

Mycotoxins are secondary metabolites produced by Aspergillus that are frequently found in spoiled agricultural commodities (such as peanuts, corns, rice, and milk), posing significant adverse health effects worldwide. ${ }^{1}$ Among them, aflatoxins (AFs) and ochratoxin A (OTA) represent the most dominant and harmful mycotoxins, ${ }^{2}$ which have been proved teratogenic, mutagenic, and carcinogenic to human beings and animals. ${ }^{3}$ There are basically four types of AFs: B1, B2, G1, and G2 and AFB1 represents the most harmful one. In many countries, legislative limits were set for AFB1 in foodstuffs. ${ }^{4}$ More importantly, AFB1 has demonstrated high chemical stability against elevated temperature through food processing, making the prevention of their entrance into the food supply chain difficult. Therefore, it is extremely important to detect their

${ }^{a}$ College of Biosystems Engineering and Food Science, Zhejiang University, 866 Yuhangtang Road, Hangzhou, 310058, China.E-mail: yanbinli@zju.edu.cn

${ }^{b}$ State Key Lab Breeding Base for Zhejiang Sustainable Plant Pest Control, Institute of Quality and Standard for Agro-products, Zhejiang Academy of Agricultural Sciences, Hangzhou 310021, China

${ }^{c}$ Department of Biological and Agricultural Engineering, University of Arkansas, Fayetteville, AR 72701, USA

$\dagger$ Electronic supplementary information (ESI) available. See DOI: 10.1039/c8ra07804a

\$ These two authors contributed equally to this work. contamination at low concentration to ensure global food safety. ${ }^{5}$ Nowadays, there are basically two methods for the detection of AFs: enzyme-linked immunoassays and chromatographic-based methods, including high performance liquid chromatography (HPLC) and immunoassay chromatography. ${ }^{6}$ The former method provides a potable and rapid method for mycotoxins detection. However, low sensitivity and complex operation procedures has hampered its practical applications. ${ }^{7}$ Chromatographic-based methods are considered as facile, sensitive and portable sensing method for AFs sensing. ${ }^{8}$ Nevertheless, this method has some drawbacks such as high-cost, technique required operation and timeconsuming sample preparation process. The incorporation of biomolecules in immunoassay chromatography also makes this method less robust. Thus, development of sensitive, low-cost and robust sensing methods for AFB1 is in high demand, especially in developing countries. ${ }^{9}$ Metal-organic frameworks (MOFs), as an emerging class of porous materials with high surface area, flexible chemical properties, rich functionalities and tuneable pore structures, have demonstrated great potential in the detection of harmful residues, especially for small molecules. ${ }^{\mathbf{1 0 , 1 1}}$ For example, a series of luminescent Zr-based highly luminescent metal-organic frameworks (LOMF) were reported as sensitive chemical sensors for toxic chemicals detection and removal, including nitroaromatic explosives. ${ }^{\mathbf{1 2 - 1 5}}$ Two water-stable 3D florescent $\operatorname{Zr}($ Iv)-based metal-organic 
frameworks were constructed with different pore structures. ${ }^{16}$ Both their florescence could be efficiently quenched with nitro compound, which enables the sensitive detection of organic explosives with the detection limit reaches as low as ppb level. 2D Cd-based LMOF was also reported for the selective detection of nitroaromatic explosives different $-\mathrm{NO}_{2}$ groups. ${ }^{17}$ Furthermore, a LMOF-based method was reported for TNP selective detection with the presence of other nitro compounds based on the specific electrostatic interaction and electron and energy transfer between TNP and LMOF. ${ }^{12}$ Taking advantage of the cation-exchange approach, a water-stable Eu-MOF was explored as sensitive and selective $\mathrm{Fe}^{3+}$ sensor with potential applications in biological system. ${ }^{18}$ However, their potential as mycotoxins sensors was seldom investigated. Li et al. reported a highly sensitive Zn-based luminescent MOF (LMOF) towards mycotoxins that achieves a low detection limit of AFB1 (46 ppb) in $10 \mathrm{~min}$. This novel sensing material is of potential for the development of applicable low-cost, rapid and sensitive mycotoxin sensors. ${ }^{19}$ However, Zn-based MOFs suffered from poor water-stability due to the fragile $\mathrm{Zn}-\mathrm{O}$ bond under the attack of $\mathrm{H}_{2} \mathrm{O}$ molecules, ${ }^{20}$ which has generally limited its applications in industrial practices, such as water purification and sensing. Here, we present a robust $\mathrm{Zr}_{6}$-cluster based water-stable LMOF termed Zr-CAU-24 with sensitive response to AFs. With waterstable $\mathrm{Zr}^{4+}$ clusters and luminescent $\mathrm{TCPB}^{4-}$ severing as second building units (SBU) and organic linkers, respectively, the prepared $\mathrm{Zr}-\mathrm{CAU}-24$ nanocrystals have demonstrated high structure stability in water due to their strong metal-ligand bond strength. ${ }^{20-23}$ As-prepared Zr-LMOF crystals not only demonstrated high surface area but also revealed improved sensitivity towards AFB1 compared to free standing $\mathrm{H}_{4} \mathrm{TCPB}$ molecules in water. The limit of detection reaches as low as $19.97 \mathrm{ppb}$ in $5 \mathrm{~min}$, which is below the applicable action level set by Food and Drug Administration (FDA) for cottonseed meals intended for beef cattle $(300 \mathrm{ppb})$ and corn and peanut products $(20 \mathrm{ppb}) .{ }^{24,25}$

\section{Results and discussion}

\section{Characterization}

Water-stable luminescent Zr-CAU-24 was synthesized according to a reported research with modifications..$^{26}$ In this study, benzoic acid was used as structure modulator to control the crystal size and morphology of prepared nanocrystals. Without benzoic acid, amorphous colloid-like polymer that is formed by the aggregation of nano-Zr-CAU-24 crystals $(\sim 30 \mathrm{~nm})$ is observed (Fig. S1 $\dagger$ ). Notably, we also found that the amorphous polymer has demonstrated negligible response towards AFB1. Thus, incorporating the $\mathrm{H}_{4}$ TCPB into the long-range order periodic structure is necessary for its sensitive response towards AFB1. The as-prepared Zr-CAU-24 crystals have demonstrated a rodlike morphology with a size of $\sim 1 \mu \mathrm{m}$ (Fig. 2A) with strong blue fluorescence. As shown in Fig. $1 \mathrm{~A}$, the $\mathrm{Zr}^{4+}$ are coordinated by eight carboxylate groups to form a C-centred orthorhombic arrangement cluster $\left(\left[\mathrm{Zr}_{6}\left(\mu_{3}-\mathrm{O}\right)_{4}\left(\mu_{3}-\mathrm{OH}\right)_{4}\right]^{12+}\right)$. The rest coordination sites at the $\mathrm{Zr}^{4+}$ ions are occupied by $\mathrm{H}_{2} \mathrm{O}$ and $\mathrm{OH}^{-}$ molecules as mentioned in other Zr-based MOFs. As reported,
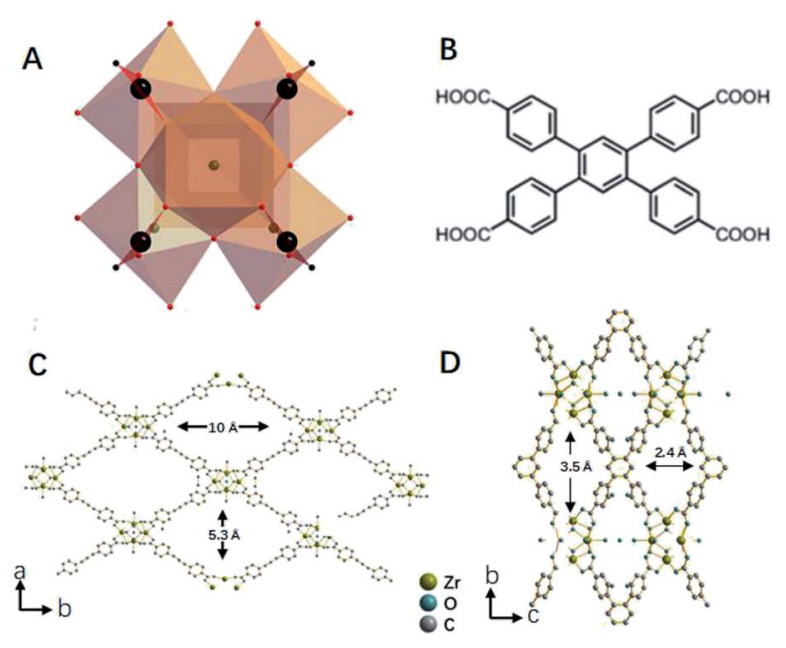

Fig. 1 Crystal structure of $\mathrm{Zr}-\mathrm{CAU}-24$ that is constructed from the $\left[\mathrm{Zr}_{6}\left(\mu_{3}-\mathrm{O}\right)_{4}\left(\mu_{3}-\mathrm{OH}\right)_{4}\right]^{12+}$ clusters (A) and $\mathrm{TCPB}^{4+}$ molecules (B). Ballstick models of $\mathrm{Zr}-\mathrm{CAU}-24$ crystals (C) and (D).

the clusters are further bridged by $\mathrm{TCPB}^{4-}$ linkers (Fig. 1B) in a SCU topology, giving rise to a porous framework with rhombic channels of $\sim 5.3 \times 10.5 \AA$ and $\sim 2.4 \times 3.5 \AA$ in diameter (Fig. 1C and D). For rapid and sensitive detection of mycotoxins in water, the structure stability of Zr-CAU-24 crystals in water is of great importance.

To prove its water-stability, the prepared Zr-CAU-24 crystals were immersed in water for $24 \mathrm{~h}$ before it is measured with Powder X-ray Diffraction (PXRD) and $\mathrm{N}_{2}$ adsorption/desorption isotherm. As shown in Fig. 2B, the XRD peaks before and after water treatment are in good agreement, indicating the intact pore structure after long time of operation in water. The PXRD pattern is also retained after the loading of AFB1, which proves that the Zr-MOF structure was well kept after its quenching of florescence by AFB1. Similar results can be found in its $\mathrm{N}_{2}$ adsorption/desorption isothermal curves before and after $24 \mathrm{~h}$
A
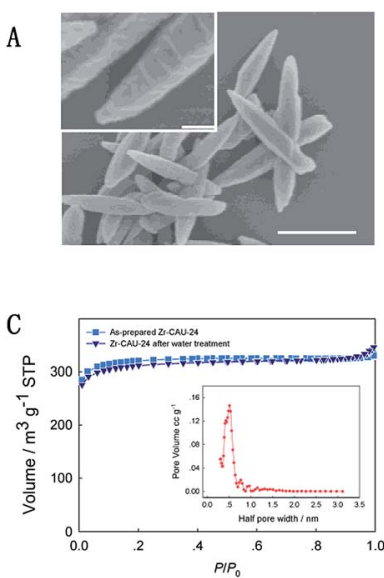

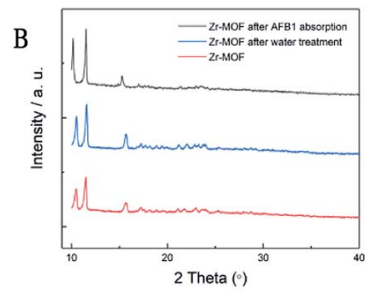

D

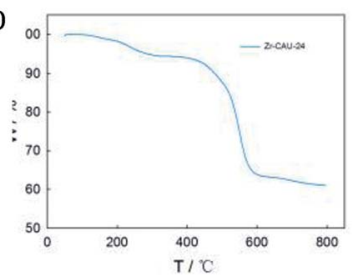

Fig. 2 Scanning electron microscopy images of prepared Zr-CAU-24 crystals with different magnification times. Scale bar: $1 \mu \mathrm{m}((A)$, inset). PXRD of Zr-CAU-24 crystals before and after water treatment and AFB1 absorption, (B) and $\mathrm{N}_{2}$ adsorption/desorption isotherms (C) of $\mathrm{Zr}$ CAU-24 crystals before and after $24 \mathrm{~h}$ water treatment. TGA result of as-prepared Zr-CAU-24 crystals (D). 
water treatment (Fig. 2C). We found that the BrunauerEmmett-Teller (BET) surface area remained, which again proves its intact pore structure during water treatment. The isothermal curve of $\mathrm{Zr}$-CAU-24 is in good agreement with type I adsorption/de-adsorption isothermal curve, which implies the uniform microporous structure of prepared Zr-CAU-24. Further pore size distribution analysis directly revealed that most of the micropores have an aperture of $1 \mathrm{~nm}$ (Fig. 2C, inset). Larger micropores of $\sim 2 \mathrm{~nm}$ with higher accessibility to mycotoxins can also be found. Thermal gravimetric analysis (TGA) was used to prove the thermal stability of Zr-CAU-24 (Fig. 2D). It first exhibited a weight loss of $3 \%$ in the temperature range $50-$ $200{ }^{\circ} \mathrm{C}$. Sequentially, further weight loss of $5 \%$ was observed between $200-420{ }^{\circ} \mathrm{C}$ (loss of internal solvent molecules). The decomposition of the thermal robust porous material starts at $420{ }^{\circ} \mathrm{C}$ with a total weight loss of $\sim 38 \%$ after degradation. With good water and thermal stability $\left(\sim 420^{\circ} \mathrm{C}\right)$, this robust sensing material is considered as an appropriate candidate for the development of low-cost, rapid and sensitive sensors for detection of mycotoxins.

\section{Detection of mycotoxins}

Mycotoxin detection was achieved by monitoring the PL signal fading of Zr-CAU-24 crystals after reacting with mycotoxins. We first determined the optimum LMOF dosage to harvest the highest FL fading by reacting with $50 \mu \mathrm{M}$ AFB1 (Fig. S2 $\dagger$ ). When $50 \mu \mathrm{g} \mathrm{mL}{ }^{-1}$ LMOF was applied, highest FL fading of $83 \%$ was achieved, while both lower and higher LMOF dosage decreased FL fading (73\%). Sequentially, we examined its performance in the detection of AFB1 by adding given amount of toxins to the solution containing $50 \mu \mathrm{g} \mathrm{mL}{ }^{-1} \mathrm{Zr}-\mathrm{CAU}-24$ crystals. The strong blue-fluorescence-emission LMOF nanocrystals were significantly quenched in $5 \mathrm{~min}$ and the signal was monitored by a fluorimeter (Fig. 3A). Under the excitation of $340 \mathrm{~nm}$
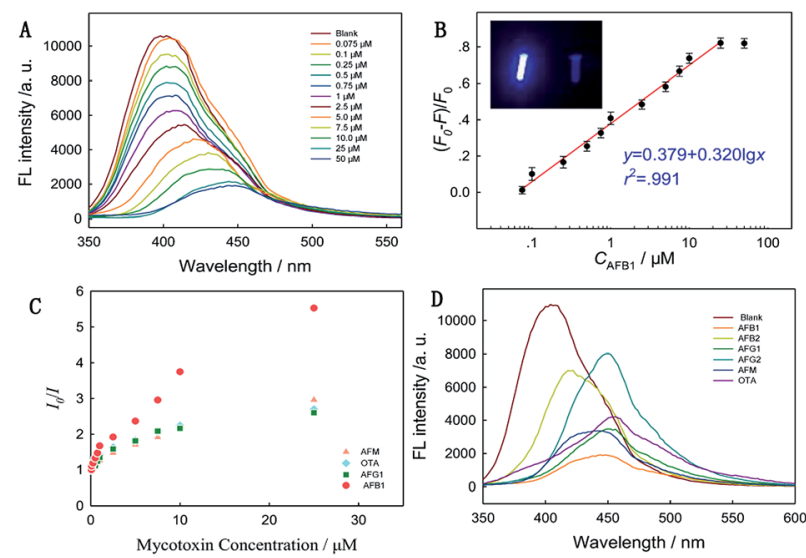

Fig. 3 Emission spectra of $\mathrm{Zr}-\mathrm{CAU}-24$ with the incremental addition of AFB1 in water, with toxin concentrations given from 0 to $50 \mu \mathrm{M}(\mathrm{A})$. Linear relationship between $\lg C_{\mathrm{AFB} 1}$ and $\mathrm{FL}$ intensity. Inset: optical photo of florescent $\mathrm{Zr}$-CAU-24 crystals in water (left) and water (right) (B). Stern-Volmer curves acquired at $\lambda_{\mathrm{ex}}=340 \mathrm{~nm}$ and $\lambda_{\mathrm{em}}=410 \mathrm{~nm}$ for AFB1, AFG1, AFM and OTA (C). FL intensity fading of Zr-CAU-24 towards $50 \mu \mathrm{M}$ AFB1, AFB2, AFG1, AFG2, AFM and OTA (D). wavelength, the fading of fluorescence intensity at $410 \mathrm{~nm}(\mathrm{Zr}-$ CAU-24) is correlated linearly with the logarithmic molar volume of toxins (eqn (1))

$$
\begin{gathered}
y=0.379+0.320 \lg x\left(r^{2}=0.991\right) \\
y=\left(F_{0}-F\right) / F_{0}
\end{gathered}
$$

where $y$ represents the relative florescence intensity, $x$ represents the molar volume of AFB1, and $F_{0}$ and $F$ is the intensity of fluorescence with and without toxins, respectively. Good linear relationship between relative fluorescence $\left(\left(F_{0}-F\right) / F_{0}\right)$ and the logarithm of toxic concentration $(>99 \%)$ is achieved for a wide range of toxin concentrations $(0.075$ to $25 \mu \mathrm{M})$, which is of advantage over its counterparts. ${ }^{27}$ The detection limit reaches as low as $19.97 \mathrm{ppb}(\mathrm{S} / \mathrm{N}=3)$. The value of this detection limit is below the tolerant level of cottonseed meals intended for beef cattle (300 ppb) and corn and peanut products (20 ppb) (FDA) and among those of the most sensitive materials reported for AFB1 sensing. Furthermore, we applied the sensing material to the detection of other two aflatoxins (AFG1, AFM) and ochratoxin A (OTA). Drastic fluorescence quenching was also observed and the liner relationships were obtained as shown in the Fig. S3-S5. $\uparrow$ They all demonstrated good linear relationship between relative fluorescence and the logarithm of toxic concentration (>98\%), ranging from 0.075 to $25 \mu \mathrm{M}$. SternVolmer quenching efficiency is used to describe its sensitivity towards different mycotoxins.

$$
I_{0} / I=K_{\mathrm{sv}}[Q]+1
$$

where $I_{0}$ represents the initial emission peak of intensity, $I$ is the emission peak intensity upon the addition of analyte, $[Q]$ is molar intensity upon the addition of analyte (quencher) and $K_{\mathrm{sv}}$ is the quenching efficiency, which is used to quantitatively evaluate the performance of Zr-CAU-24 as a mycotoxin sensor. As shown in Fig. 3C, this porous nanomaterial has demonstrated sensitive quenching effect towards four kinds of mycotoxins including AFB1, AFG1, AFM and OTA and the $K_{\mathrm{sv}}$ reaches 138 461, 50 793, 68 119, $53149 \mathrm{M}^{-1}$, respectively. The $K_{\mathrm{sv}}$ for AFB1 is almost doubled compared with that for AFG1, AFM and OTA, indicating its higher selectivity toward AFB1. Stronger orbital overlap ( $\pi-\pi$ conjugation) between AFB1 and Zr-CAU-24 is reported responsible for the enhanced selectivity towards AFB1 compared with other kinds of mycotoxins. ${ }^{14}$ To better reveal the different responses of $\mathrm{Zr}-\mathrm{CAU}-24$ toward different mycotoxins, we treated the analyst with a fixed concentration $(50 \mu \mathrm{M})$ of AFB1, AFB2, AFG1, AFG2, AFM and OTA (Fig. 3D). The fluorescence intensity at $380 \mathrm{~nm}$ dropped drastically after adding of mycotoxins. Obvious red shift was observed in all test samples, which is due to the fluorescent properties of mycotoxins under the excitation of $340 \mathrm{~nm}$.

Walnut and almond beverages are common products made from nuts, exposing them to the risk of being contaminated by AFB1. Therefore, we further applied this sensing material to the direct detection of AFB1 in the spiked samples (Table 1). AFB1 of three different concentrations $(0.1 \mu \mathrm{M}, 1 \mu \mathrm{M}, 10 \mu \mathrm{M})$ were 
Table 1 Recovery rates of AFB1 in spiked walnut and almond beverages using fluorescent Zr-CAU-24 crystals (HPLC method was used as comparison)

\begin{tabular}{lcllr}
\hline & Spiked amount $(\mu \mathrm{M})$ & HPLC $(\mu \mathrm{M})$ & Proposed method $(\mu \mathrm{M})$ & \multicolumn{1}{c}{ Recovery $(\%)$} \\
\hline Walnut beverage & 0.1 & 0.102 & 0.0975 & $98 \%$ \\
& 1.0 & 1.007 & 1.079 & $98 \%$ \\
Almond beverage & 10.0 & 9.969 & 9.73 & $97 \%$ \\
& 0.1 & 0.0946 & 0.0966 & $97 \%$ \\
& 1.0 & 0.956 & 1.041 & 9.06 \\
\end{tabular}

tested with $\mathrm{Zr}$-CAU-24 and HPLC method is valued as a control to evaluate the recovery rates in real samples (Fig. S7†). High recoveries were achieved in all samples (91-108\%), which is comparable to that of HPLC (95-102\%). At low toxin concentration $(0.1 \mu \mathrm{M})$, the recovery rates for walnut and almond beverages both reached $93 \%$. It's noticed that this sensing method has demonstrated a wide liner range of $0.075-25 \mu \mathrm{M}$, while most of reported methods have narrow liner ranges. ${ }^{28-30}$ These results have revealed the potential of this water-stable sensing material for the development of low-cost, rapid, and potable mycotoxin sensors.

\section{Mechanism of mycotoxin detection}

By anchoring the fluorophore into the framework, the absorption wavelength presents a red shift from $280 \mathrm{~nm}$ to $330 \mathrm{~nm}$ (Fig. 4A) due to the strong $\pi-\pi$ interaction in the framework, which is observed in other sensing materials reported. However, the emission wavelength of $\mathrm{TCPB}^{4-}$ remained at $410 \mathrm{~nm}$ (Fig. 4B), which implies a higher energy transfer efficiency in Zr-CAU-24 crystals. $\mathrm{TCPB}^{4-}$ is a strong fluorophore, but it has no strong interaction with AFs (Fig. 4C). Also, nano-Zr-CAU-24 crystals of $\sim 30 \mathrm{~nm}$ demonstrates negligible fluorescence quenching effect towards AFB1. Thus, incorporating the fluorophore into a long-range order structure of metal-organic framework is essential for sensitive and selective quenching of AFB1. The quenching mechanism of LMOF as a mycotoxin sensor was due to the electron transfer between the LMOF and mycotoxins because the LUMO energy state of MOF is above the LUMO state of mycotoxins. ${ }^{19}$ However, the enhanced quenching efficiency of anchored $\mathrm{TCPB}^{4-}$ molecules in the $\mathrm{Zr}-\mathrm{CAU}-24$ was barely discussed. $\mathrm{N}_{2}$ absorption/desorption curve demonstrates that the average pore size slightly decreases from $1 \mathrm{~nm}$ to $0.8 \mathrm{~nm}$, which demonstrates that most pores of Zr-MOF is not occupied after AFB1 adsorption. Combined with the absorption testing result (Fig. S6 $\dagger$ ), it is found that the AFB1 was fully absorbed by the Zr-CAU-24 crystals and removed from the solution after sensing and the notable enhancement may be due to the amplified luminescence quenching of luminescent MOFs at the surface. The periodic structure of phosphorescent $\mathrm{Zr}$-CAU24 has allowed for long distance transfer of intra-MOF energy, which significantly enhances the electron-transfer quenching of fluorescence Zr-CAU-24 at the surface ${ }^{31}$ and thereby causes higher quenching efficiency of micron level Zr-CAU-24 crystals compared with nano-Zr-CAU-24 of $\sim 30 \mathrm{~nm}$.
A

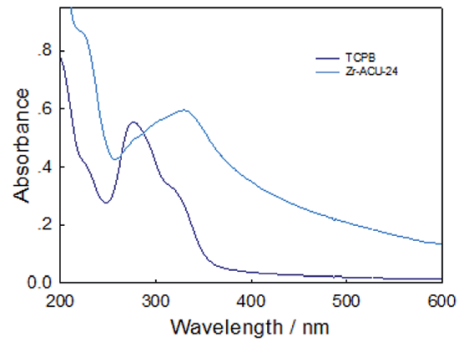

B
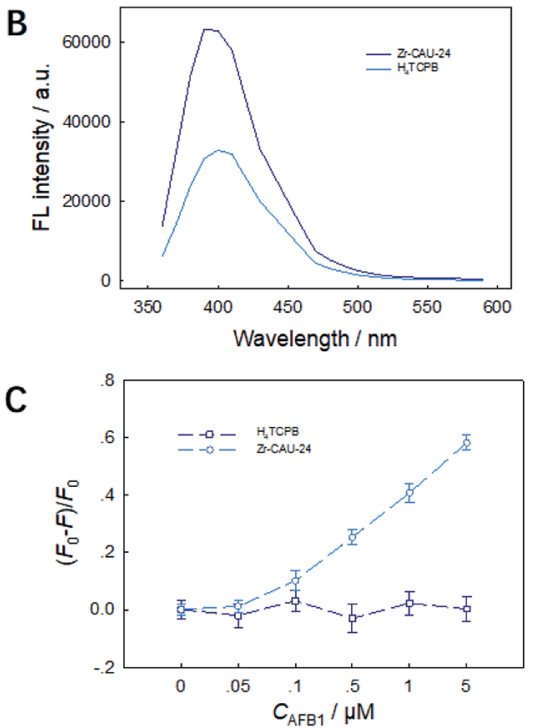

Fig. 4 UV-visible spectrum (A) and emission spectra $\left(\lambda_{\mathrm{em}}=410 \mathrm{~nm}\right)$ (B) of $\mathrm{TCPB}^{4+}$ molecules and $\mathrm{Zr}-\mathrm{CAU}-24$ crystals. FL intensity fading of $\mathrm{Zr}-\mathrm{CAU}-24$ crystals and $\mathrm{TCPB}^{4+}$ molecules with the existence of AFB1 (C).

\section{Experimental}

\section{Instrumentation}

Hitachi SU-8000 field-emission electron scanning microscope (Hitachi, Japan) was used to characterize the morphology and the EDS of MOF particles with an acceleration voltage of $3 \mathrm{kV}$ (10 nm gold coating). Powder X-ray Diffraction was conducted on an $\mathrm{X}^{\prime}$ Pert PRO diffractometer (PANalytical, Netherland) equipped with $\mathrm{Cu} \mathrm{K} \alpha$ radiation $(\lambda=0.15406 \mathrm{~nm}$ ) (scan speed: $1^{\circ} \min ^{-1} ; 2 \theta$ range: $2-50^{\circ}$; step size: $0.05^{\circ}$ ). The BET surface area of the MOF particles was measured using an AUTOSORB-1-C surface area and pore size analyzer (Quantachrome, USA) with samples degassed at $200{ }^{\circ} \mathrm{C}$ for $24 \mathrm{~h}$ in advance. The thermal 
stability of the MOF particles was determined on a Pyris 1 thermal gravimetric analyzer (Perkin Elmer, USA) with a ramp rate of $10^{\circ} \mathrm{min}^{-1}$ up to $600{ }^{\circ} \mathrm{C}$. DU $800 \mathrm{UV}$-visible spectrometer (Beckman Coulter, USA) with $1 \mathrm{~cm}$ quartz cuvettes was used to measure the absorbance of $\mathrm{TCPB}^{4-}$ and $\mathrm{Zr}-\mathrm{CAU}-24$. High Performance Liquid Chromatography (HPLC, Agilent 1260 Infinity, USA) was used to accurately determine the AFB1 concentration in both walnut and almond beverages. Analytical balance with an accuracy of $0.01 \mathrm{mg}$ (Mettler Toledo, USA) was used.

\section{Reagents}

Zirconium chloride (97\%), $\mathrm{H}_{4} \mathrm{TCPB}$ (99\%) and benzoic acid (98\%) were purchased from Sigma-Aldrich (USA). AFB1, AFB2, AFG1, AFG2, AFM, OTA, methanol (97\%) and arsenic acid (80\%) were purchased from Sinopharm Chemical Reagent Co., Ltd (Shanghai, China). Walnut and almond beverages were purchased from a local supermarket. All other chemicals were of analytical grade or better quality and used as received. Milli-Q ultrapure water (Millipore, USA; $\geq 18 \mathrm{M} \Omega \mathrm{cm}$ ) was used throughout.

\section{Synthesis of Zr-CAU-24}

Zr-CAU-24 was prepared according to a reported article with

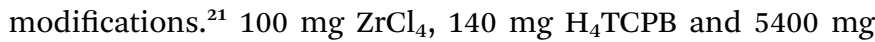
benzoic acid were ultrasonically dissolved in $32 \mathrm{~mL} \mathrm{H}_{2} \mathrm{O}$ : DMF $(1: 2)$ in a $50 \mathrm{~mL}$ Teflon vessel. The clear mixture was then kept in reaction kettle at $120{ }^{\circ} \mathrm{C}$ and maintained for $48 \mathrm{~h}$. After cooling down to room temperature, the obtained white cloudy liquid was washed with DMF once and methanol twice and dried at $60{ }^{\circ} \mathrm{C}$ overnight. For the preparation of amorphous colloid-like polymer, $50 \mathrm{mg}$ of $\mathrm{H}_{4}$ TCPB was allowed to react with $70 \mathrm{mg}$ of $\mathrm{ZrCl}_{4}$ in $8 \mathrm{~mL}$ of dimethyl formamide in a Teflon vessel baker for $24 \mathrm{~h}$ at $120{ }^{\circ} \mathrm{C}$ before washed with methanol for 3 times. The product was dried at $60^{\circ} \mathrm{C}$ overnight.

\section{AFB1 absorption}

$100 \mu \mathrm{M}$ AFB1 and $0.5 \mathrm{mg} \mathrm{mL}^{-1} \mathrm{Zr}$-MOF were mixed for $10 \mathrm{~min}$ before being centrifugated at $8000 \mathrm{rpm}$ for $10 \mathrm{~min}$. The supernate was collected and measured using a fluorimeter.

\section{Fluorescence detection}

Typically, $4 \mathrm{mg}$ of Zr-CAU-24 were simply dispersed in $10 \mathrm{~mL}$ of water at room temperature. The mixture was oscillated for $2 \mathrm{~min}$ to prepare the metal ion incorporated suspension for luminescent measurements. Then $90 \mu \mathrm{L}$ of diluted $\mathrm{Zr}-\mathrm{CAU}-24$ $\left(55 \mu \mathrm{g} \mathrm{mL}^{-1}\right)$ aqueous solution was added with $10 \mu \mathrm{L}$ of AFB1 solution with final concentrations of $0.075,0.1,0.25,0.5$, $0.75,1.0,2.5,5.0,7.5,10,25$, and $50 \mu \mathrm{M}$. For specificity test, Zr-CAU-24 was mixed with $50 \mu \mathrm{M}$ AFs (OTA, AFB1, AFB2, AFG1, AFG2, or AFM) or with $10 \mu \mathrm{L}$ of water as a blank control. For the test on mechanism of mycotoxin detection, $90 \mu \mathrm{L}$ of $\mathrm{H}_{4}$ TCPB $\left(55 \mu \mathrm{g} \mathrm{mL} \mathrm{m}^{-1}\right)$ aqueous solution was added with $10 \mu \mathrm{L}$ of AFB1 solution with final concentrations of 0.05 , $0.1,0.5,1.0$ and $5 \mu \mathrm{M}$.

\section{Analysis of AFB1 in spiked samples}

In this study, walnut and almond beverages without sprayed AFs were used for spiked experiments. The obtained spiked samples $(5 \mathrm{~mL})$ were added with $1 \mathrm{~g}$ of $\mathrm{NaCl}$ and then dissolved into $25 \mathrm{~mL}$ of methanol-water solution (volume ratio: methanol : $\mathrm{H}_{2} \mathrm{O}=7: 3$ ). The mixed solution was filtrated and purified by immunoaffinity chromatography column, finally filtrated by $0.22 \mu \mathrm{m}$ filter membrane before chromatographic determination.

\section{Conclusions}

We have presented a facile method for rapid and sensitive detection of microscale mycotoxins using a water-stable porous Zr-based luminescent MOF. It has demonstrated ultrasensitive response towards AFB1 with a detection limit of $19.97 \mathrm{ppb}$ in 5 min (from sample detection to signal output) which is below the applicable action level set by FDA for cottonseed meals intended for beef cattle (300 ppb) and corn and peanut products (20 ppb). The quenching efficiency for AFB1 was as high as $138461 \mathrm{M}^{-1}$, which is almost a doubled value compared to that of AFG1, AFM and OTA, which indicates a higher selectivity for AFB1. Due to the good water-stability of prepared LMOF, we further applied the prepared Zr-CAU-24 without further modification to the detection of toxins in spiked samples of walnut and almond beverages. High recovery rates $(\geq 91 \%)$ were achieved in the detection of different concentrations of AFB1. The amplified quenching effect of TCPB-based MOFs compared with unanchored TCPB $^{4-}$ molecules and nano-Zr-CAU-24 may be due to the amplified quenching on the surface of $\mathrm{Zr}-\mathrm{CAU}-24$ crystals. Thus, we have presented a simple, cost-saving, robust and sensitive method for the detection of AFB1 with acceptable detection limit and time, which has great potential for the development of innovative sensors for detection of mycotoxins in agriculture and food.

\section{Conflicts of interest}

There are no conflicts to declare.

\section{Acknowledgements}

This research was supported in part by the National Natural Science Foundation of China (Grants 31671940), Zhejiang Key Research and Development Project (2015C02041) and the National Key Technology Research and Development Program of the Ministry of Science and Technology of China (No. 2013BAD19B02).

\section{References}

1 S. Marin, A. J. Ramos, G. Cano-Sancho and V. Sanchis, Food Chem. Toxicol., 2013, 60, 218-237.

2 S. Z. Iqbal, T. Rabbani, M. R. Asi and S. Jinap, Food Chem., 2014, 157, 257-262. 
3 M. Edite Bezerra da Rocha, F. da C. O. Freire, F. Erlan Feitosa Maia, M. Izabel Florindo Guedes and D. Rondina, Food Control, 2014, 36, 159-165.

4 F. and A. O. of the U. Nations, FAO Food and Nutrition Paper 81, 2004.

5 A. G. Marroquin-Cardona, N. M. Johnson, T. D. Phillips and A. W. Hayes, Food Chem. Toxicol., 2014, 69, 220-230.

6 S. Yue, X. Jie, L. Wei, C. Bin, W. Dou Dou, Y. Yi, L. QingXia, L. JianLin and Z. TieSong, Anal. Chem., 2014, 86, 1179711802.

7 L. Yang and R. Bashir, Biotechnol. Adv., 2008, 26, 135-150.

8 N. V. Beloglazova, E. S. Speranskaya, A. Wu, Z. Wang, M. Sanders, V. V. Goftman, D. Zhang, I. Y. Goryacheva and S. De Saeger, Biosens. Bioelectron., 2014, 62, 59-65.

9 G. S. Shephard, Food Addit. Contam., Part A: Chem., Anal., Control, Exposure Risk Assess., 2008, 25, 146-151.

10 Z. Hu, B. J. Deibert and J. Li, Chem. Soc. Rev., 2014, 43, 58155840.

11 H. Furukawa, K. E. Cordova, M. O'Keeffe and O. M. Yaghi, Science, 2013, 341, 1230444.

12 S. S. Nagarkar, B. Joarder, A. K. Chaudhari, S. Mukherjee and S. K. Ghosh, Angew. Chem., Int. Ed. Engl., 2013, 52, 2881-2885.

13 S. S. Nagarkar, A. V Desai and S. K. Ghosh, Chem. Commun., 2014, 50, 8915-8918.

14 S. Xie, H. Wang, Z. Liu, R. Dai and L. Huang, RSC Adv., 2015, 5, 7121-7124.

15 N. S. Bobbitt, M. L. Mendonca, A. J. Howarth, T. Islamoglu, J. T. Hupp, O. K. Farha and R. Q. Snurr, Chem. Soc. Rev., 2017, 46, 3357-3385.

16 B. Wang, X.-L. Lv, D. Feng, L.-H. Xie, J. Zhang, M. Li, Y. Xie, J.-R. Li and H.-C. Zhou, J. Am. Chem. Soc., 2016, 138, 62046216.

17 S. R. Zhang, D. Y. Du, J. S. Qin, S. J. Bao, S. L. Li, W. W. He, Y. Q. Lan, P. Shen and Z. M. Su, Chem.-Eur. J., 2014, 20, 3589-3594.
18 S. Dang, E. Ma, Z.-M. Sun and H. Zhang, J. Mater. Chem., 2012, 22, 16920-16926.

19 Z. Hu, W. P. Lustig, J. Zhang, C. Zheng, H. Wang, S. J. Teat, Q. Gong, N. D. Rudd and J. Li, J. Am. Chem. Soc., 2015, 137, 16209-16215.

20 H. Jasuja, N. C. Burtch, Y. G. Huang, Y. Cai and K. S. Walton, Langmuir, 2013, 29, 633-642.

21 N. C. Burtch, H. Jasuja and K. S. Walton, Chem. Rev., 2014, 114, 10575-10612.

22 J. H. Cavka, S. Jakobsen, U. Olsbye, N. Guillou, C. Lamberti, S. Bordiga and K. P. Lillerud, J. Am. Chem. Soc., 2008, 130, 13850-13851.

23 D. Feng, Z.-Y. Gu, J.-R. Li, H.-L. Jiang, Z. Wei and H.-C. Zhou, Angew. Chem., 2012, 124, 10453-10456.

24 USFDA, CPG Sec. 570.375 Aflatoxin in Peanuts and Peanut Products, 2015.

25 USFDA, CPG Sec. 683.100 Action Levels for Aflatoxins in Animal Feeds, 2015.

26 M. Lammert, H. Reinsch, C. A. Murray, M. T. Wharmby, H. Terraschke and N. Stock, Dalton Trans., 2016, 45, 18822-18826.

27 Y. Xu, B. Chen, Q. He, Y. Lou Qiu, X. Liu, Z. He and Z. Xiong, Anal. Chem., 2014, 86, 8433-8440.

28 W. Xu, Y. Xiong, W. Lai, Y. Xu, C. Li and M. Xie, Biosens. Bioelectron., 2014, 56, 144-150.

29 J. C. Vidal, L. Bonel, A. Ezquerra, S. Hernández, J. R. Bertolín, C. Cubel and J. R. Castillo, Biosens. Bioelectron., 2013, 49, 146-158.

30 S. Piermarini, L. Micheli, N. H. S. Ammida, G. Palleschi and D. Moscone, Biosens. Bioelectron., 2007, 22, 1434-1440.

31 C. A. Kent, D. Liu, T. J. Meyer and W. Lin, J. Am. Chem. Soc., 2012, 134, 3991-3994. 